\title{
Dynamic and static structure factor of electrons in Si: Inelastic x-ray scattering results
}

\author{
W. Schülke, J. R. Schmitz, H. Schulte-Schrepping, and A. Kaprolat \\ Institut für Physik, Universität Dortmund, D-44221 Dortmund, Germany
}

(Received 31 March 1995; revised manuscript received 10 July 1995)

\begin{abstract}
The dynamic structure factor $S(\mathrm{q}, \omega)$ of electrons in single-crystal Si was measured with 1.6-eV resolution by means of inelastic $x$-ray scattering spectroscopy for $q \|$ [100], [110], and [111] with $0.37<q<2.06$ a.u. using synchrotron radiation from the DORIS storage ring. By utilizing the $f$-sum rule, $S(\mathbf{q}, \boldsymbol{\omega})$ could be brought on to an absolute scale, so that also the static structure factor $S(q)$ could be obtained. The orientation-averaged features of the dynamic and the static structure factor, such as dispersion, width, and shape of the spectra, could be brought in reasonable agreement with jellium calculation, when we went beyond the random-phase approximation by taking into account both exchange corrections via a static local-field factor and momentum-dependent lifetime in an on-shell approximation of the self-energy. Results of fitting of the static local-field factor to the experiment are presented. The rich qorientation-dependent fine structure found in the experimental $S(\mathbf{q}, \boldsymbol{\omega})$ spectra either could be attributed to the enhanced density of states on zone boundaries in the extended zone scheme, or could be understood in terms of plasmon-Fano resonances, which are the result of a plasmon-band-induced coupling between the continuum of electron-hole excitations and the discrete plasmon resonances. This interpretation is supported by pseudopotential calculations within the limits of the two-plasmon-band model.
\end{abstract}

\section{INTRODUCTION}

It has recently been shown how synchrotronradiation-based inelastic $x$-ray scattering spectroscopy (IXSS) provides valuable information about dynamic properties of electrons in $s-p$ metals and $s-p$ semiconductors, ${ }^{1-6}$ where the energy resolution and statistical accuracy could be made much higher than that achieved in pioneering experiments, ${ }^{7-9}$ which used conventional $x$ ray sources. Thus many lattice-induced effects could be found in the dynamic structure factor $S(\mathrm{q}, \omega)$, a quantity which can be measured on an absolute scale by means of IXSS, and which is the Fourier transform in space and time of the ground-state expectation value of the timedependent density-density correlation operator. Latticeinduced effects include the so-called zone-boundary collective states (ZBCS's), identified in IXSS results for $\mathrm{Li}$ (Ref. 1) and $\mathrm{Be},{ }^{10}$ and the plasmon-Fano resonances found in IXSS Specta of Si. ${ }^{11}$ Moreover, by utilizing the potentials of coherent inelastic X-ray scattering, ${ }^{12-14}$ we have presented experimental evidence of the volume - plasmon-band structure in $\mathrm{Si}$, and have obtained estimates of the magnitude of the plasmon-band gap ${ }^{15}$ and the shape of the bands near the Brillouin-zone boundary. ${ }^{16}$

The very promising IXSS results for $\mathrm{Si}$ gave rise to a more systematic IXSS investigation of $\mathrm{Si}$, presented in what follows, where the aim of these measurements was not only to look more systematically at lattice-induced effects, but also to make a careful analysis of whether the experimental data may give evidence of a breakdown of the (exchange-corrected) random-phase approximation (RPA). Such a breakdown was claimed in the mid-1970s by several authors, ${ }^{17,18}$ since their electron-energy-loss spectroscopy (EELS) results for $\mathrm{Al}$ and Si seemed to indicate that the plasmon energy became nearly constant for $q>q_{c} \quad\left(q_{c}\right.$ is the plasmon cutoff vector). Conversely,
Chen, Meixner, and Kincaid ${ }^{19}$ obtained an overall agreement of their EELS data for $\mathrm{Si}$ with an (exchangecorrected) homogeneous electron-gas RPA calculation. The tremendous difficulties of measuring $S(\mathrm{q}, \omega)$ with EELS for larger $q$ 's $\left(q>q_{c}\right)$, due to the strong contamination of the spectra by multiple-scattering events, make the EELS results questionable for $q>q_{c}$, so that conclusions drawn from these results remain a matter of debate. Therefore, we decided to look carefully into the dispersion of the $S(\mathrm{q}, \omega)$ peaks of $\mathrm{Si}$, especially for larger $q$ 's. Theoretical predictions for the dynamic structure factor of Si known so far are restricted to the plasmon range $\left(q<q_{c}\right){ }^{20-22}$ For that reason we have supported our conclusions, drawn from experimental results for $q>q_{c}$, by a model calculation of the dielectric response of Si taking into account local-field effects via the full dielectric matrix. ${ }^{23-25}$

Deviations from the RPA should not only be recognizable in the dynamic structure factor, but also in the static structure factor $S(q)$, whose Fourier transform, the radial distribution function $g(r)$, exhibits a strong unphysical negative dip at $r=0$, when calculated within the limits of the RPA. Therefore, we have utilized the fact that we can measure the dynamic structure factor on an absolute scale (by means of the $f$-sum rule), so that it is not difficult to obtain the static structure factor by energy integration.

This paper is organized as follows. In Sec. II we present some fundamental relations for the dielectric response of solids, inasmuch as they will be utilized for discussing the experimental results and for performing model calculations. The experimental setup is described in Sec. III. Section IV presents experimental results obtained for $S(\mathrm{q}, \omega)$ and $S(\mathrm{q})$, which are discussed in Sec. $\mathrm{V}$ on the basis of model calculations, where we go beyond the RPA and take into account the full dielectric matrix. Final conclusions are drawn in Sec. VI.

11721

(c) 1995 The American Physical Society 


\section{BASIC RELATIONS}

First-order perturbation theory directly relates the double-differential scattering cross section (DDSCS) $d^{2} \sigma / d \omega d \Omega$ for inelastic x-ray scattering to the dynamic structure factor $\mathbf{S}(\mathbf{q}, \omega) \mathrm{via}^{26}$

$$
d^{2} \sigma / d \omega d \Omega=\left(\mathbf{e}_{0} \cdot \mathbf{e}^{\prime}\right)^{2} r_{0}^{2}\left(\omega^{\prime} / \omega_{0}\right) \mathbf{S}(\mathbf{q}, \omega),
$$

where $\hbar \omega_{0}$ and $\hbar \omega^{\prime}$ are the energies, $\mathbf{e}_{0}$ and $\mathbf{e}^{\prime}$ are the polarization vectors of the incident and the scattered photons, respectively, $\hbar \omega$ is the energy transferred to the scattering system, $\mathrm{q}$ is the transferred momentum, and $r_{0}$ is the classical electron radius. The dynamical structure factor $S(\mathrm{q}, \omega)$ is the Fourier transform in time and space of the ground-state expectation value of the timedependent density-density correlation operator $n_{2}\left(\mathbf{r}^{\prime}, \mathbf{r}, t\right)$ :

$$
n_{2}\left(\mathbf{r}^{\prime}, \mathbf{r}, t\right) \equiv\left\langle\mathbf{I}\left|\sum_{j, j^{\prime}} \delta\left[\mathbf{r}-\mathbf{r}_{j}(0)\right] \delta\left[\mathbf{r}^{\prime}+\mathbf{r}-\mathbf{r}_{j^{\prime}}(t)\right]\right| \mathbf{I}\right\rangle,
$$

where the summation is over the electrons of the scattering system:

$$
\begin{aligned}
S(\mathbf{q}, \omega)=\iiint & n_{2}\left(\mathbf{r}^{\prime}, \mathbf{r}, t\right) \exp (-i \omega t) \\
& \times d t \exp (-i \mathbf{q} \cdot \mathbf{r}) d \mathbf{r} d \mathbf{r}^{\prime} .
\end{aligned}
$$

The fluctuation-dissipation theorem ${ }^{27}$ connects the dynamical structure factor with the dielectric response function $\varepsilon^{-1}(\mathbf{q}, \omega)$ :

$$
S(\mathrm{q}, \omega)=-\left(\hbar q^{2} / 4 \pi^{2} e^{2} n\right) \operatorname{Im} \varepsilon^{-1}(\mathrm{q}, \omega),
$$

where $n$ is the electron density.

According to the local-field dielectric theory of Adler ${ }^{23}$ and Wiser, ${ }^{24}$ the dielectric response function can be expressed in terms of a diagonal element of the inverse dielectric matrix:

$$
\begin{aligned}
\varepsilon^{-1}(\mathbf{q}, \omega) & =\left(\varepsilon_{\mathbf{G}^{\prime} \mathbf{G}^{\prime \prime}}\left(\mathbf{q}_{r}, \omega\right)\right)_{\mathrm{GG}}^{-1} \\
& =\left(\delta_{\mathbf{G}^{\prime} \mathbf{G}^{\prime \prime}}+T_{\mathbf{G}^{\prime} \mathbf{G}^{\prime \prime}}\right)_{\mathrm{GG}}^{-1} .
\end{aligned}
$$

$\mathbf{G}, \mathbf{G}^{\prime}$, and $\mathbf{G}^{\prime \prime}$ are reciprocal-lattice vectors, where $\mathbf{G}$ reduces $q$ into the first Brillouin zone according to $\mathbf{q}=\mathbf{q}_{r}+\mathbf{G}$. Within the limits of the self-consistent-field theory, ${ }^{28}$ which is equivalent to the RPA, the matrix $T_{\mathrm{G}^{\prime} \mathrm{G}^{\prime \prime}}$ is given by

$$
\begin{array}{r}
T_{\mathbf{G}^{\prime} \mathbf{G}^{\prime \prime}}=\left[4 \pi e^{2} /\left(\mathbf{q}_{r}+\mathbf{G}^{\prime}\right)^{2}\right] \lim _{\eta \rightarrow 0} \sum_{\substack{\mathbf{k}, v \\
\mathbf{k}^{\prime}, v^{\prime}}}\left\langle\mathbf{k} \boldsymbol{v}\left|\exp \left[-i\left(\mathbf{q}_{r}+\mathbf{G}^{\prime}\right) \cdot \mathbf{r}\right]\right| \mathbf{k}^{\prime} v^{\prime}\right\rangle\left\langle\mathbf{k}^{\prime} v^{\prime}\left|\exp \left[i\left(\mathbf{q}_{r}+\mathbf{G}^{\prime \prime}\right) \cdot \mathbf{r}\right]\right| \mathbf{k} v\right\rangle \\
\times\left[f_{0}\left(\mathbf{k}^{\prime}, v^{\prime}\right)-f_{0}(\mathbf{k}, v)\right] /\left[\hbar \omega+E(\mathbf{k}, v)-E\left(\mathbf{k}^{\prime}, v^{\prime}\right)+i \eta\right]
\end{array}
$$

using the one-electron representation with Bloch states $|\mathbf{k}, v\rangle$, energy $E(\mathbf{k}, v)$, and occupation number $f_{0}(\mathbf{k}, v)$, where $\mathbf{k}$ is the reduced wave vector and $\boldsymbol{v}$ the band index. If we neglect all local-field effects, the dielectric matrix becomes diagonal, so that we obtain the well-known Ehrenreich-Cohen ${ }^{28}$ dielectric function

$$
\varepsilon(\mathbf{q}, \omega)=1+\lim _{\eta \rightarrow 0}\left(4 \pi e^{2} / q^{2}\right) \sum_{\substack{\mathbf{k}, v \\ \mathbf{k}^{\prime}, v^{\prime}}}\left|\left\langle\mathbf{k}^{\prime}, v^{\prime}|\exp (i \mathbf{q} \cdot \mathbf{r})| \mathbf{k}, v\right\rangle\right|^{2}\left[f_{0}\left(\mathbf{k}^{\prime}, v^{\prime}\right)-f_{0}(\mathbf{k}, v)\right] /\left[\hbar \omega+E(\mathbf{k}, v)-E\left(\mathbf{k}^{\prime}, v^{\prime}\right)+i \eta\right] .
$$

Our model calculation, presented in what follows, went two steps beyond the RPA.

(1) We introduced the finite lifetime of electron-hole excitations by adding

$$
i\left[\Gamma\left(\mathbf{k}^{\prime}, v^{\prime}\right)+\Gamma(\mathbf{k}, v)\right]
$$

to the denominator of Eqs. (6) and (7), respectively, where $\Gamma(\mathbf{k}, v)$ is approximated by the modulus of the imaginary part of the on-shell self-energy $\Sigma(p)$ of the corresponding homogeneous electron system:

$\Gamma(\mathbf{k}, v)=|\operatorname{Im} \Sigma(p)| \equiv \Gamma(p), \quad p=(2 m E(\mathbf{k}, v))^{1 / 2}$.

$\Sigma(p)$ can be calculated using a scheme proposed by Quinn and Terrell. ${ }^{29}$ This way we neglect the off-shell contributions to the self-energy, a procedure introduced by Rahman and Vignale ${ }^{30}$ in order to explain the double-peak structure of the dynamical structure factor. Although this approximation has been criticized by $\mathrm{Ng}$ and $\mathrm{Da}$ browski, ${ }^{31}$ it should, at least qualitatively, account for the most important effect of finite lifetime on the dynamic structure factor, namely the steep rise of $\Gamma(p)$ at a certain critical momentum $p_{0}$, where $p_{0}$ is the minimum quasiparticle momentum which makes possible the decay of the quasiparticle into a plasmon:

$$
\hbar \omega\left(q_{c}\right)=\left(\hbar^{2} / 2 m\right)\left(p_{0}^{2}-k_{F}^{2}\right),
$$

with the plasmon energy $\hbar \omega\left(q_{c}\right)$ at the plasmon cutoff vector $q_{c}$ and the Fermi momentum $k_{F}$. Adding the onshell self-energies to the energy denominator in Eq. (7) is tantamount to a relaxation-time approximation that fails to conserve the local electron number. We have removed this defect in our model calculation of Sec. V A by using Mermin's ${ }^{32}$ formalism.

(2) We have exchange corrected ${ }^{11}$ the response function of Eq. (5) by utilizing the static approximation $\boldsymbol{G}(q)$ of the so-called local-field factor $G(\mathrm{q}, \omega)$ (Ref. 33) in

$\varepsilon_{\mathbf{G}^{\prime} \mathbf{G}^{\prime \prime} \mathrm{corr}}(\mathbf{q}, \omega)=\delta_{\mathrm{G}^{\prime} \mathbf{G}^{\prime \prime}}+(1-G(\mathbf{q}, \omega)) T_{\mathbf{G}^{\prime} \mathbf{G}^{\prime \prime}}(\mathbf{q}, \omega)$

and

$$
\begin{aligned}
\left(\operatorname{Im} \varepsilon^{-1}(\mathbf{q}, \omega)\right)_{\mathrm{corr}}=- & \operatorname{Im}\left[\left(\varepsilon_{\mathbf{G}^{\prime} \mathbf{G}^{\prime \prime} \text { corr }}\left(\mathbf{q}_{r}, \omega\right)\right)_{\mathbf{G G}}^{-1}\right] \\
& \times[1 /(1-G(\mathbf{q}, \omega))] .
\end{aligned}
$$

We have used the numerical values of $G(q)$ from Ichimaru and Utsumui. ${ }^{34}$ 


\section{EXPERIMENT AND DATA PROCESSING}

The DDSCS of $\mathrm{Si}$ was measured at the Hamburger Synchrotronstrahlungslabor (HASYLAB) using synchrotron $x$ rays from the wiggler HARWI ("Harter Röntgen Wiggler"), installed at the storage ring DORIS. The experimental station is described in detail elsewhere. ${ }^{35}$ The major part of the horizontal divergence of the radiation emitted by the wiggler is accepted by the first watercooled plane (511) Si crystal, and is then sagittally focused to $8-\mathrm{mm}$ horizontal width onto the scattering sample by the second cylindrically bent (511) Si monochromator crystal, which was segmented in order to prevent anticlastic bending. ${ }^{36}$ The flux of useful $13.7-\mathrm{keV}$ photons in a focus of $8 \times 8 \mathrm{~mm}^{2}$ at the sample position was $3.8 \times 10^{12} \mathrm{~s}^{-1}$, with the DORIS storage ring operated at $3.7 \mathrm{GeV}$ and $60 \mathrm{~mA}$. The energy analysis of the scattered radiation has been performed in inverse geometry using a (1200) spherically bent Si crystal, set to a fixed Bragg angle of $86^{\circ}$ by varying the primary energy, where dispersion compensation ${ }^{37}$ has been fully utilized. The overall energy resolution obtained was $1.6 \mathrm{eV}$. The effective diameter $D=4 \mathrm{~cm}$ of the analyzer crystal and the sampleto-analyzer distance defines an error $\Delta q=0.04$ a.u. (a.u. means atomic units with $\hbar=e=m=1$ ) of the momentum transfer. The sample of $0.6-\mathrm{mm}$ thickness has been investigated in transmission geometry.

The Si data were processed as described in detail in Ref. 2: The count rate of the detector at the exit slit of the analyzer is related to the count rate of a detector which monitors the beam leaving the double-crystal monochromator, so that only relative values of the spectral intensity are measured. After subtraction of both a linear background (the relative spectral intensity at $\hbar \omega=-10 \mathrm{eV})$ and the quasielastically scattered line (which is assumed to be symmetric, so that its lowenergy-loss side can be mirror reflected to produce reliable values of its high-energy-loss side), the relative values of the DDSCS were brought to an absolute scale by means of the $f$-sum rule. ${ }^{2}$ It must be mentioned that the way we subtracted the quasielastically scattered line produces a range of uncertainty between $0-$ and $5-\mathrm{eV}$ energy loss. Whenever $q$ is within a range of strong thermal diffuse scattering, which means that $q$ is near a reciprocal-lattice vector $\mathbf{G}$, the quasielastically scattered line can be larger than the spectrum of the inelastically scattered radiation by more than two orders of magnitude, so that a reliable subtraction of the quasielastically scattered line turned out to be hopeless. This is the reason why the IXSS measurements of $S(q, \omega)$, as presented below, for certain values of $q$, do not contain all principal directions of $q$. Between $1 \times 10^{3}$ and $2 \times 10^{3}$ counts were collected in each channel at the peaks of the spectra, which took between 10 and $20 \mathrm{~min}$. In order to focus attention on the main features of the spectral fine structure, the spectra were smoothed by means of cubic splines, where the algorithm used ${ }^{38}$ additionally provides the first three derivatives. In Fig. 1, the result of smoothing of the Si q\|[111]; $q=1.25$ a.u. spectrum is exemplarily shown. In order to compare our IXSS results with the corresponding EELS data ${ }^{18,19}$ and with theoretically cal-

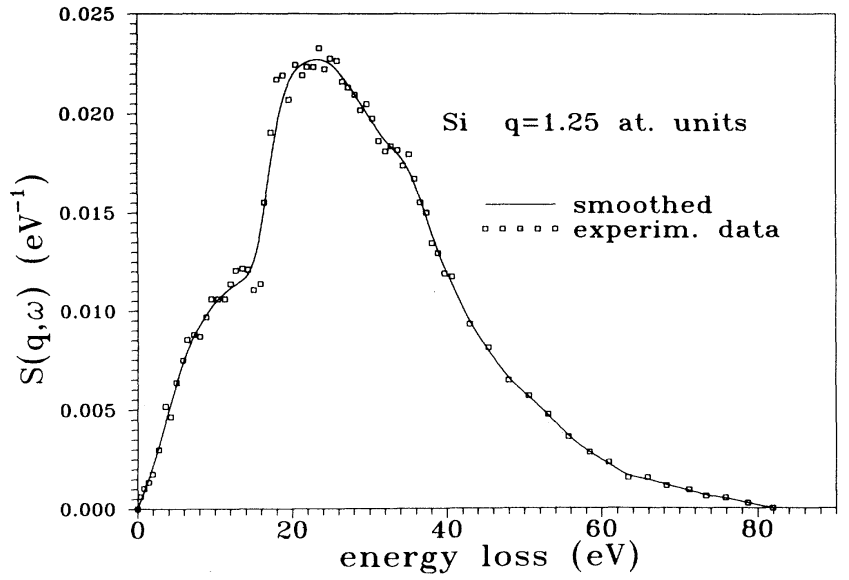

FIG. 1. Circles: Experimental data of $S(\mathbf{q}, \omega)$ of $\mathrm{Si}$ for $\mathbf{q}$ parallel to [111] and $q=1.25$ a.u., after background subtraction, removal of the quasielastically scattered line, and scaling to absolute values by utilizing the $f$-sum rule. Solid line: Cubic spline interpolation of the experimental data.

culated dispersion relations, the position of the main peak of the $S(\mathbf{q}, \omega)$ spectra has to be defined in a unique manner. We have chosen the following center of gravity method for this purpose: If $I_{\max }$ was the absolute maximum value of the $S(q, \omega)$ spectrum, we defined the peak position $\hbar \omega_{\text {peak }}$ by

$$
\begin{aligned}
& \omega_{\text {peak }}=\frac{1}{21} \sum_{v=0}^{20} \frac{1}{2}\left[\omega_{>}\left(I_{v}\right)+\omega_{<}\left(I_{v}\right)\right], \\
& I_{v}=\left[\frac{1}{2}+(v / 60)\right] I_{\max },
\end{aligned}
$$

where $\hbar \omega_{>}\left(I_{v}\right)$ and $\hbar \omega_{<}\left(I_{v}\right)$ are the energy losses corresponding to $I_{v}$ on the high- and low-energy-loss flanks of the $S(\mathrm{q}, \omega)$ spectrum, respectively.

Since we obtained $S(\mathbf{q}, \omega)$ on an absolute scale, it was also possible to obtain the static structure factor $S(q)$ of the Si valence electrons by integration with respect to $\omega$, according to

$$
S(\mathbf{q})=\int_{0}^{\infty} S(\mathbf{q}, \omega) d \omega
$$

The most reliable values of $S(q)$ have been obtained for $q$ 's, where the measured spectrum has already reached the linear background level for $\omega<\omega_{L}$, where $\hbar \omega_{L}$ is the energy of the Si $L_{\text {III }}$ edge $(99.2 \mathrm{eV})$, the onset of core contributions to $S(\mathrm{q}, \omega)$. This was true for $q \leq 1.78$ a.u. In the remaining case $(q=2.02$ a.u.), we have extrapolated the valence electron contribution by fitting the spectrum to an exponential, starting $10 \mathrm{eV}$ below the $L_{\mathrm{III}}$ edge. This procedure has also been used in the process of absolute scaling of the spectra for $q>1.78$ a.u. Therefore, a larger error has to be attributed to $S(q)$ for $q>1.78$ a.u. Moreover, for the same reason it does not seem to be significant to aspire to experimental data of $S(q)$ for $q$ much larger than 2.0 a.u., though this is the $q$ range of $S(q)$ around $2 k_{F}$, which is of special interest for a correlated Fermi liquid. 


\section{SURVEY OF EXPERIMENTAL RESULTS}

In Figs. 2-5, measured $S(\mathrm{q}, \omega)$ spectra of $\mathrm{Si}$ for $\mathrm{q}$ parallel to [100], [110], and [111], respectively, within a range of $0.37 \leq q \leq 2.02$ a.u., are presented, smoothed by means of cubic splines. The spectra exhibit, especially for larger $q$ 's, a rich fine structure, far beyond experimental error. The fine structure, and to a certain extent also the peak position, depend on the $q$ orientation. Even for smaller $q$ 's, additional peaks (shoulders) appear (see, for example, the [100] spectrum for $q=0.59$ a.u.), which have not been observed in the EELS spectra of Refs. 18 and 19. The main peak position of the IXSS $S(\mathbf{q}, \omega)$ spec-

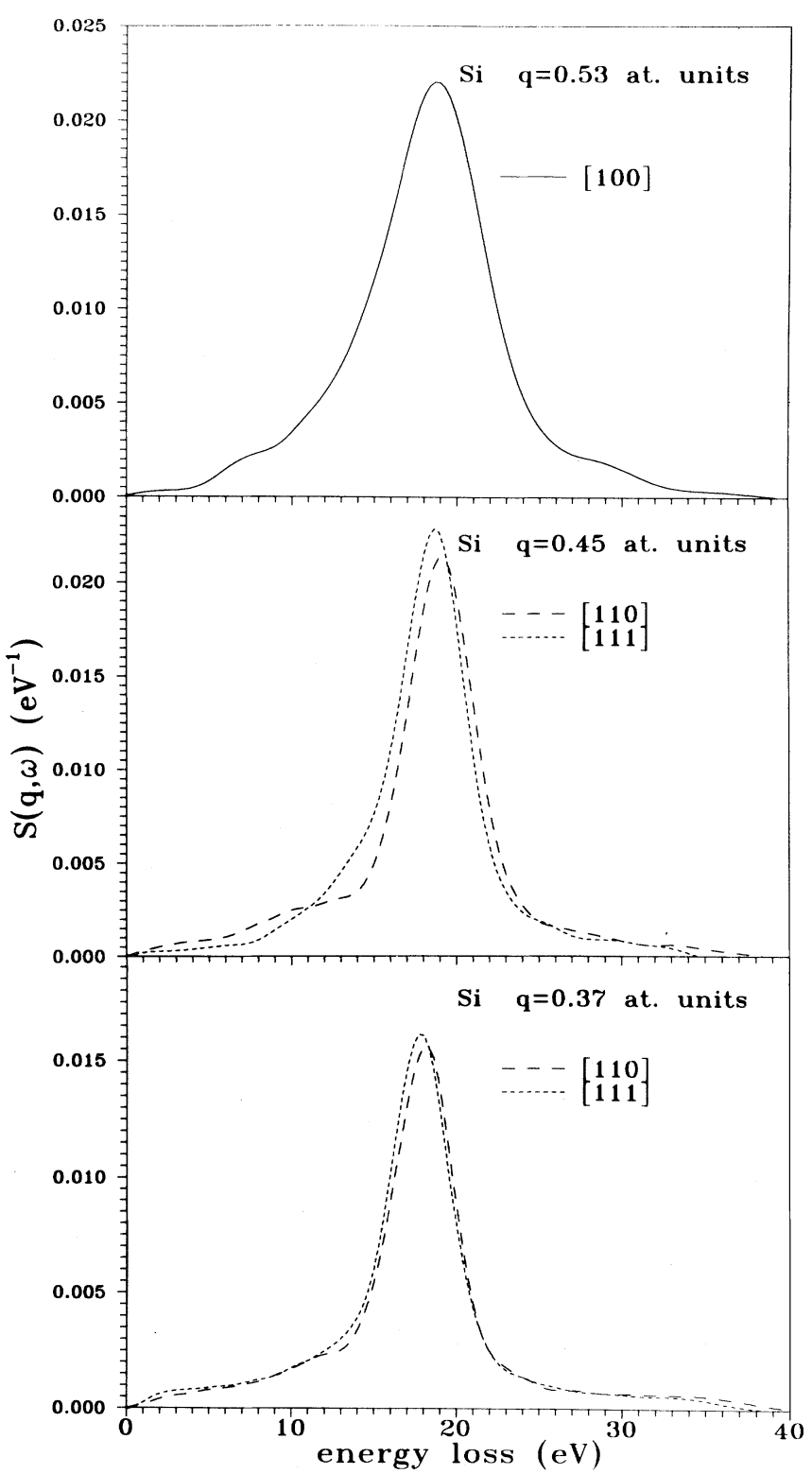

FIG. 2. Cubic-spline-interpolated experimental $S(\mathbf{q}, \omega)$ spectra of Si for $q=0.37,0.45$, and 0.53 a.u., and $q$ orientations as indicated.

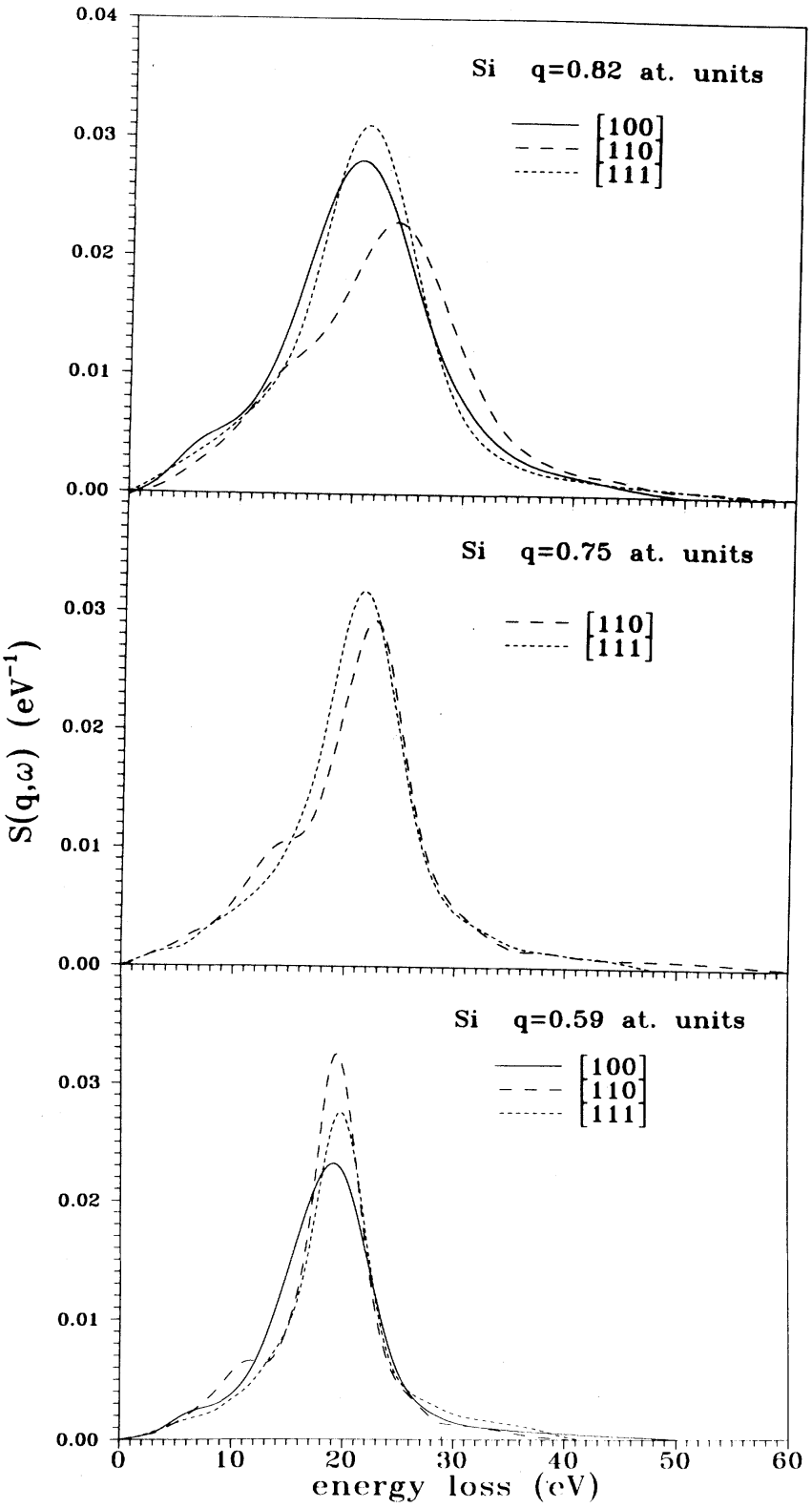

FIG. 3. Cubic-spline-interpolated experimental $S(\mathbf{q}, \omega)$ spectra of Si for $q=0.59,0.75$, and 0.82 a.u., and $\mathrm{q}$ orientations as indicated.

tra are plotted in Fig. 6 as a function of $q$, together with the peak position in the corresponding EELS results of Refs. 18 and 19, respectively. As can be derived from Fig. 6, the orientation-averaged dispersion for $q<1.0$ a.u. of the respective main peak of the experimental IXSS $S(\mathrm{q}, \omega)$ spectra is quite consistent with the EELS data of both Refs. 18 and 19. For 1.0 a.u. $<q<1.3$ a.u., the IXSS peak positions agree better with the data of Ref. 19, so that the tendency to zero dispersion, as found in the EELS data of Ref. 18, could not be verified. The IXSS $S(\mathbf{q}, \omega)$ data for $q>1.3$ a.u. are unique, since for these large $q$ 's EELS measurements are overloaded with 


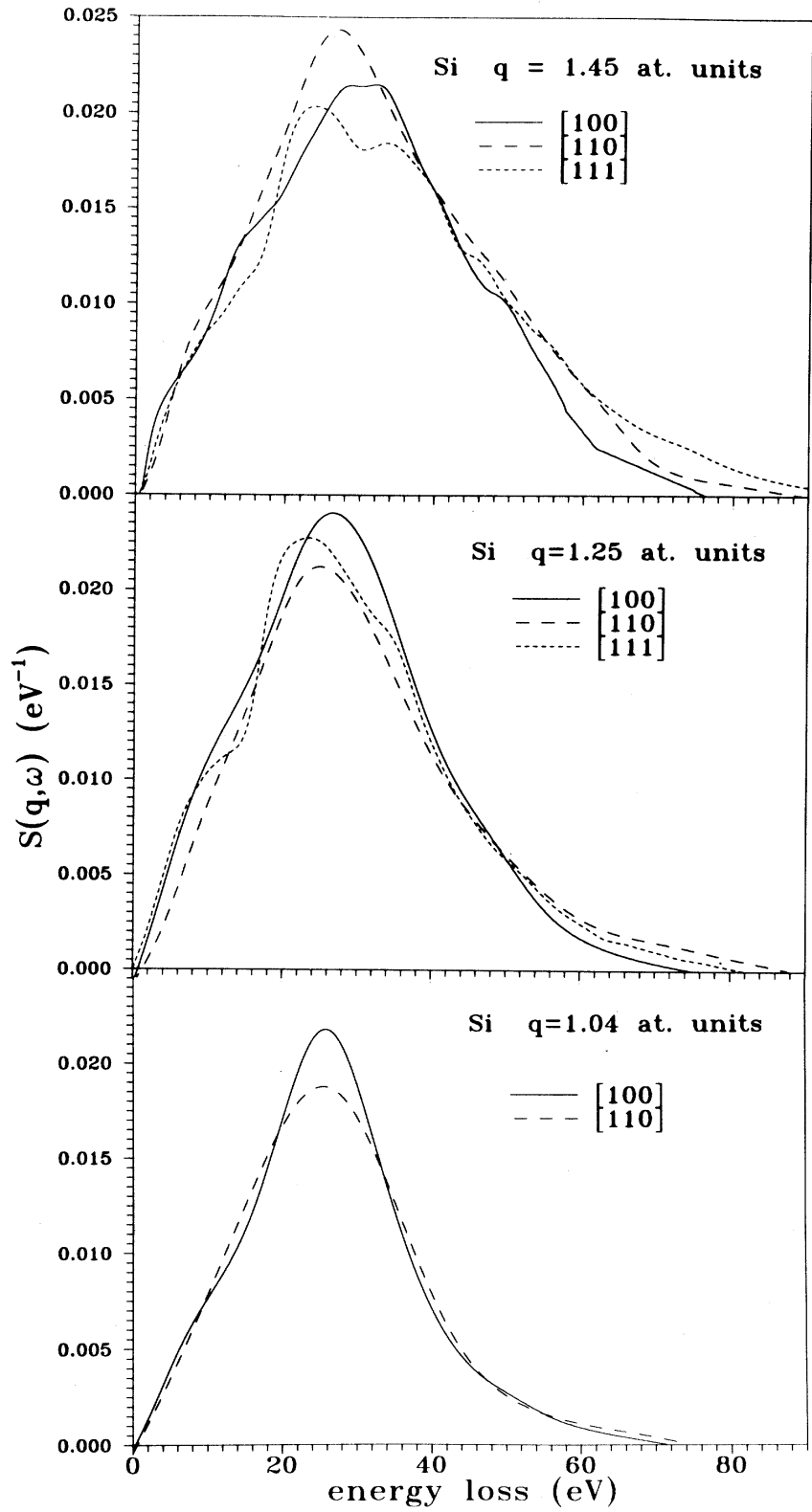

FIG. 4. Cubic-spline-interpolated experimental $S(\mathbf{q}, \omega)$ spectra of Si for $q=1.04,1.25$, and 1.45 a.u., and $q$ orientations as indicated.

multiple-scattering features, which are difficult to correct for. In this $q$ range, one observes a strong dispersion of the peak position of $S(\mathbf{q}, \omega)$ after relative weak dispersion between 0.8 and 1.4 a.u. In some cases, the peak position of $S(\mathbf{q}, \omega)$ is anisotropic, where there exists no clear order of the peak positions with respect to the principal directions, even for $q$ 's smaller than the corresponding freeelectron-plasmon cutoff vector $q_{c}=0.63 \mathrm{a}$.u. of $\mathrm{Si}$.

In contrast to simple metals like $\mathrm{Al},{ }^{6}$ the plasmonlike resonances of $\mathrm{Si}$ for $q<q_{c}$ are much broader; their full width at half maximum (FWHM), as averaged over the

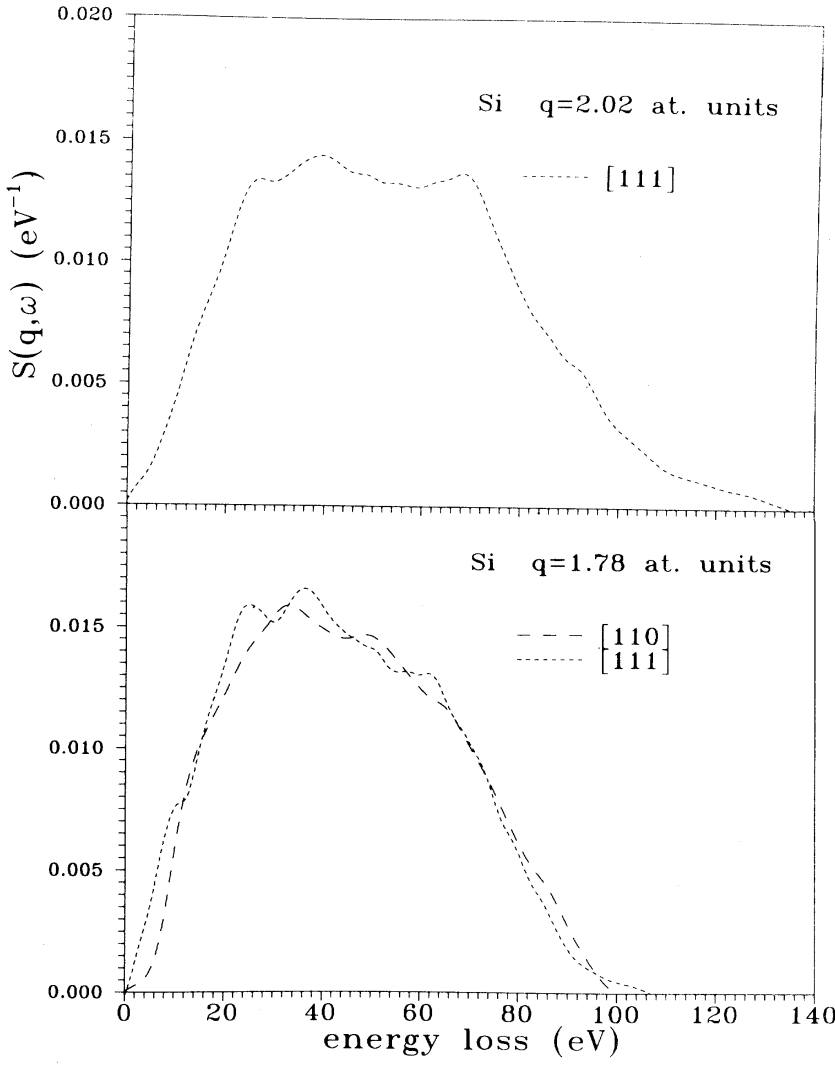

FIG. 5. Cubic-spline-interpolated experimental $S(\mathbf{q}, \omega)$ spectra for $q=1.78$ and 2.02 a.u., and $q$ orientations as indicated.

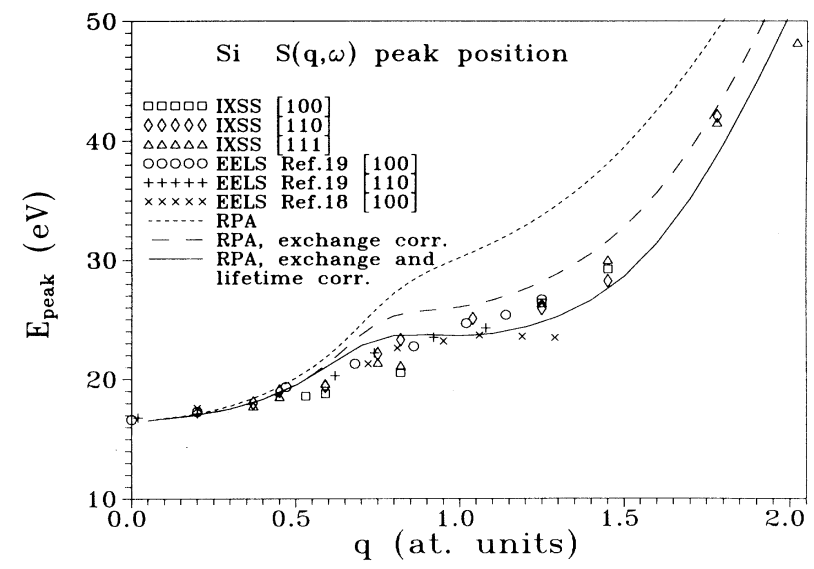

FIG. 6. Dispersion of the Si $S(\mathbf{q}, \omega)$ peak positions of the IXSS experiment for q parallel to [100], [110], and [111], respectively, as indicated in the inset; dispersion of the $\mathrm{Si} S(\mathbf{q}, \omega)$ peak position of the EELS experiment of Refs. 18 and 19 for $q$ orientations as indicated in the inset. Dispersion of the jelliummodel-calculated Si $S(\mathbf{q}, \omega)$ peak position for different approximations as indicated in the inset. 
two q orientations [110] and [111], ranges from $4.4 \mathrm{eV}$ for $q=0.37$ to $5.8 \mathrm{eV}$ for $q=0.59$ a.u. The FWHM of the spectrum for $\mathrm{q} \|[100], q=0.59$ a.u., is still much larger, namely $9.2 \mathrm{eV}$. The width of the $S(\mathrm{q}, \omega)$ spectra is not rapidly increasing, as in the case of simple metals, ${ }^{6}$ when one goes from the (free electron) plasmon excitation range $\left(q<q_{c}\right)$ to the electron-hole-pair excitation range $\left(q>q_{c}\right)$.

The shape of the $S(\mathbf{q}, \omega)$ spectra for $q<q_{c}$ is highly asymmetric in such a way that the low-energy-loss tail is much more extended than the high-energy-loss tail. For q $\|[110]$, a clear secondary peak with positive dispersion arises within the low-energy-loss tail in the range 0.59 a.u. $\leq q \leq 1.45$ a.u., which is most pronounced for $q=0.59$ a.u., and which will be attributed in Sec. V B to an enhanced density of states near the (220) Bragg plane. For larger $q$ 's $(q>1.0$ a.u.), the asymmetry of the spectra turns around, so that the tail on the high-energy-loss range is now much more extended than that on the lowenergy-loss side. For $q>1.7$ a.u., the shapes of the spectra are mainly characterized by a broad plateau, which drops off in the direction of higher-energy losses.

For all $q$ 's, especially for $q>q_{c}$, the spectra exhibit a large q-orientation dependence as far as their fine structure is concerned. The most prominent example is the peak/shoulder around $\hbar \omega=14 \mathrm{eV}$ in the [111] spectra together with the extremely steep rise between 17 and 19 eV, when compared with the [100] and [110] spectra, respectively. This structure will be discussed in terms of so-called plasmon-Fano resonances ${ }^{11}$ in Sec. V C.

In Fig. 7, the static structure factor $S(\mathrm{q})$, as derived by energy integration of the dynamic structure factor $S(\mathbf{q}, \omega)$, is presented, where the experimental error, plotted at the orientation average of $S(q)$, is composed both of the statistical error of the $S(\mathrm{q}, \omega)$ measurement and of the scaling error, which results from applying the $f$-sum rule. The latter source of error is mainly responsible for

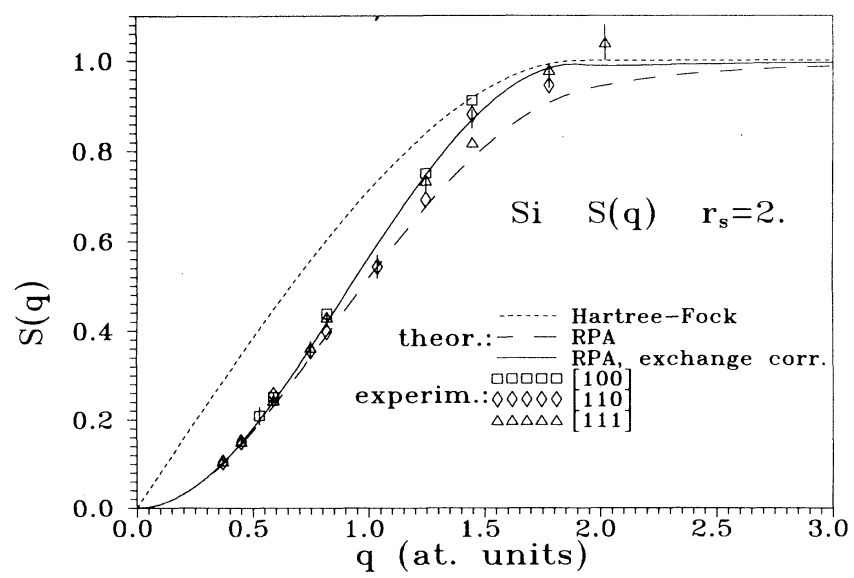

FIG. 7. Static structure factor $S(q)$ of electrons in $\mathrm{Si}$ obtained from the IXSS experiment for q parallel to [100], [110], and [111], respectively, as indicated in the inset. The error bars refer to the orientation average. Jellium-model-calculated static structure factor $S(q)$ of electrons in Si for different approximations as indicated in the inset. the relative large error at $q=2.02$ a.u., since in that case the application of the $f$-sum rule needs the elimination of the core contribution by extrapolation (see Sec. III). In one case $(q=1.45$ a.u. $), S(q)$ exhibits a q-orientation dependence which is outside the experimental error.

\section{DISCUSSION OF RESULTS, COMPARISON WITH MODEL CALCULATIONS}

\section{A. Shape and dispersion of $S(q, \omega)$ spectra, jellium model calculations}

In order to come to a consistent physical interpretation of the experimental $S(\mathbf{q}, \omega)$ spectra, we started width jellium model calculations of different degrees of approximation. The results of these calculations were compared with experimental spectra obtained by averaging directional spectra for each $q$, taking into account their different weights.

As a first step, we used the response function $\varepsilon^{-1}(\mathbf{q}, \omega)$ of Eq. (5) for an empty lattice in Eq. (4), where a small energy width for all one-electron states of 0.03 a.u. has been allowed. This procedure is equivalent with utilizing the well-known Lindhard's analytical jellium-RPA dielectric function. In Fig. 8 these calculations are compared with experiment for three different exemplary $q$ values with $q>q_{c}$. It is evident, and has already been found for $\mathrm{Li}^{2}$ $\mathrm{Be},{ }^{5}$ and $\mathrm{Al},{ }^{6}$ that there is a distinct discrepancy between calculation and experiment for all three $q$ values. In all cases, the peak position of the RPA calculation is at much larger energy losses, and the overall shapes of the calculated spectra are much more asymmetric than the experimental ones. This fact is also demonstrated in Fig. 6 , where the experimental (main) peak positions are compared with the jellium-RPA-calculated peak positions, which are far from experiment, except for the calculated data for $q \leq 0.45$ a.u., which agree quite well with experiment as far as the peak position is concerned. Even for this $q$ range, the jellium-RPA calculation cannot account for the relatively large widths of the experimental spectra. In the next step of the approximation, we have used the exchange-corrected dielectric matrix of Eq. (12) for an empty lattice, where the static approximation $G(q)$ of the so-called local-field factor $G(q, \omega)$, as calculated by Ichimaru and Utsumi ${ }^{34}$ and shown for the electron density of $\mathrm{Si}\left[r_{s}=(3 / 4 \pi n)^{1 / 3}=2\right]$ in Fig. 9, has been applied. One can see that this correction shifts the peak position in the right direction. A further step to an overall agreement between model calculation and experiment, as far as the general shape and the peak position are concerned, could be achieved by introducing, in addition to the exchange correction, an energy- (momentum-) dependent lifetime in the sense of an on-shell approximation of the imaginary part of the self-energy $\Gamma(p)$, as pointed out in Sec. II. The self-energy for the electron density of $\mathrm{Si}$ $\left(r_{s}=2\right)$ has been calculated by means of a scheme, proposed by Quinn and Terrel, ${ }^{29}$ and shown in Fig. 10. As can be seen in both Figs. 6 and 8, the addition of a lifetime correction to the exchange-corrected jellium RPA leads to a better overall agreement between model calculation and experiment. For example, even the most im- 


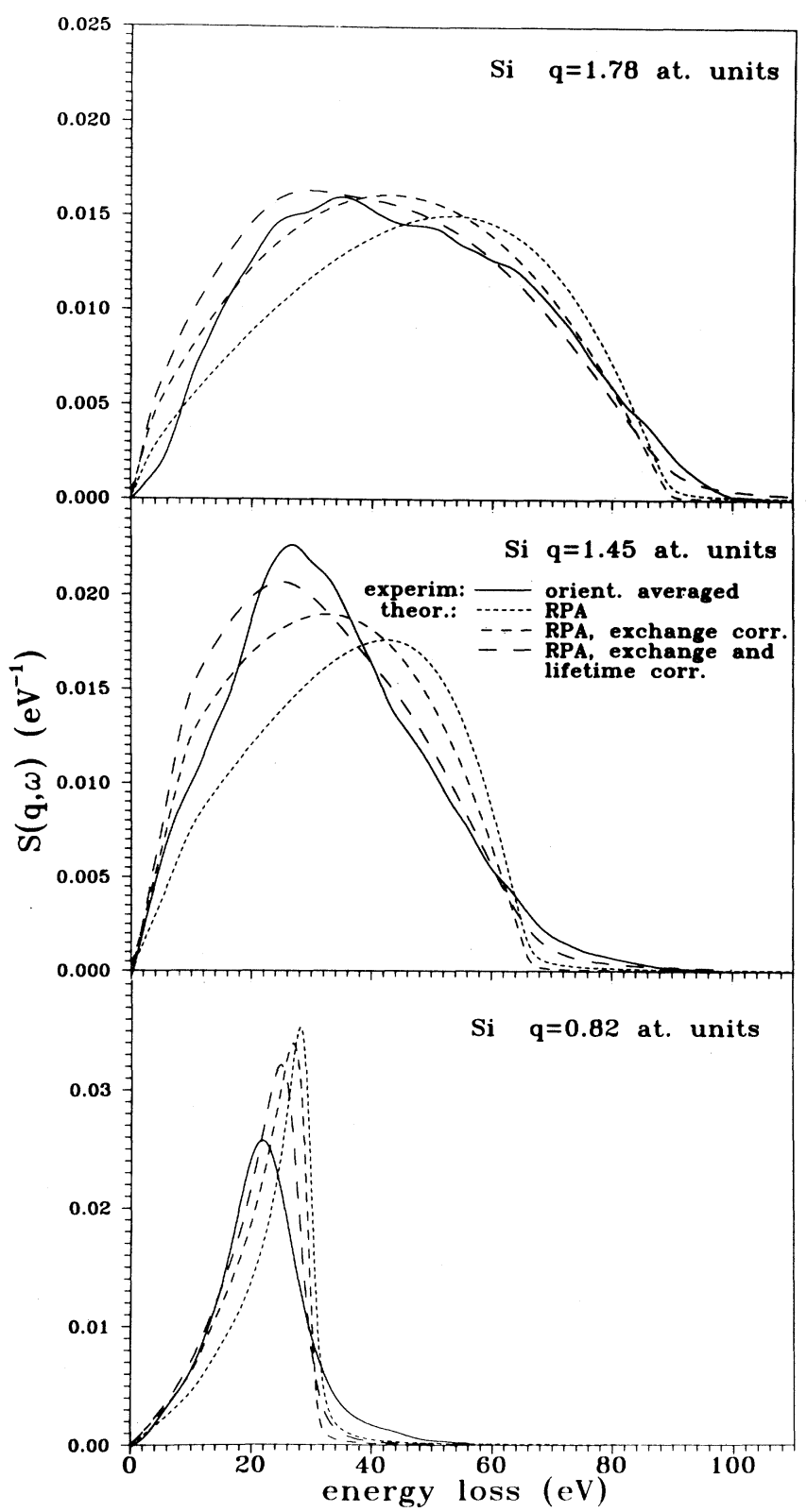

FIG. 8. Solid line: Cubic-spline-interpolated experimental dynamic structure factor $(\mathbf{q}, \omega)$ of $\mathrm{Si}$, q orientation averaged for $q$ values as indicated. Dashed curves: jellium-model-calculated $S(\mathbf{q}, \omega)$ curves for different approximations as indicated in the inset.

portant characteristic of the overall shape of the experimental spectra for $q=1.78$ a.u. [Fig. 5(c)], namely both an asymmetry, which is opposite to the typical asymmetry of uncorrected RPA spectra, and a broad plateau, which drops in the direction of increasing energy losses, are well reproduced by the calculation. To some extent, this is also true for $q=1.45$ a.u.

Looking more closely at Fig. 6, one can realize that, for 0.5 a.u. $<q<1.0$ a.u., the corrections applied to the RPA are not sufficient to shift the peak position in such a way that complete agreement with the experiment could

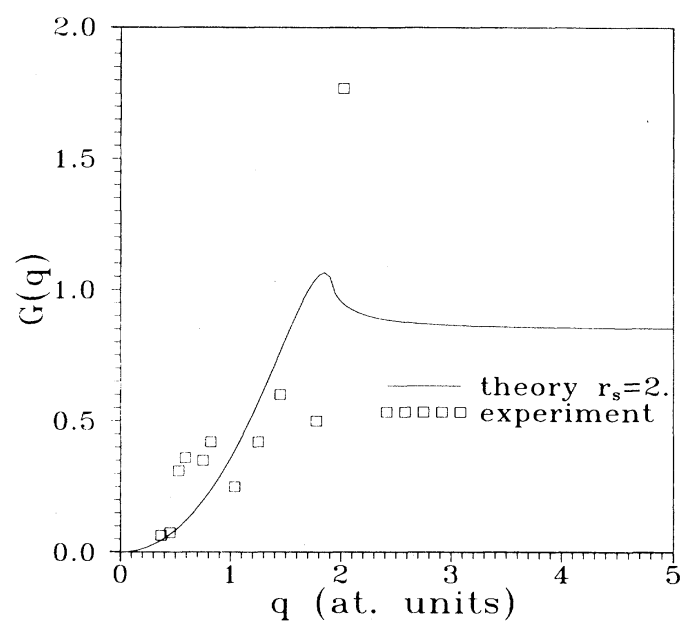

FIG. 9. Solid line: Local-field correction factor $G(q)$ of jellium for the electron density of $\mathrm{Si}, r_{s}=2$, according to an analytical expression of Ref. 34. Squares: Experimental values of $G(q)$ for $\mathrm{Si}$, obtained by fitting the peak position of orientationaveraged experimental $S(\mathbf{q}, \omega)$ 's to theoretical ones (lifetime and exchange-correlated jellium RPA), where $G(q)$ has been varied.

be achieved. On the contrary, for 1.0 a.u. $<q<1.8$ a.u., the RPA is overcorrected, whereas the correction is again far from sufficient for the $q=2.02$ a.u. spectrum. One might be tempted to attribute this behavior to a wrong choice of the so-called local-field factor $G(q)$, especially since this choice among other published $G(q)$ 's was rather arbitrary. Therefore, we have tried to find experimental values of $G(q)$ that fit best the model-calculated peak position of $S(\mathbf{q}, \omega)$ to the orientation-averaged experiment, where both the experimental and calculated peak positions are defined according to $\mathrm{Eq}$ (13). The result of this fitting procedure is plotted in Fig. 9, and exhibits quite an unusual behavior, especially around $q=k_{F}$

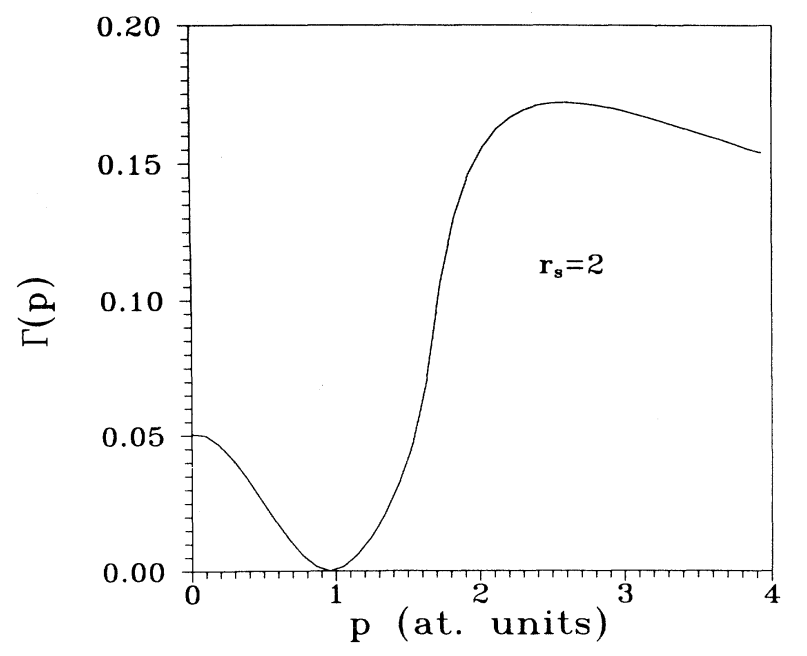

FIG. 10. On-shell approximation of the imaginary part $\Gamma(p)$ of the self-energy of jellium for the electron density of $\mathrm{Si}, r_{s}=2$. 
$\left(k_{F}=0.96\right.$ a.u. for $\left.r_{s}=2\right)$. Moreover, the enhancement of $G(q)$ above 1 near $q=2 k_{F}$ is much more pronounced in the experiment than in the theoretical values that we have chosen, although this statement is supported by only one measured value. It should be stressed that there exist other theoretical calculations, for instance in Ref. 39 , where $G(q)=2$ near $q=2 k_{F}$. Nevertheless, the results of this fitting procedure should not be overestimated, since lattice effects could be of rather large importance. We will evaluate recent $S(\mathbf{q}, \omega)$ measurements on Li (Ref. 2) and Al (Ref. 6) in the same manner, add more measurements near $q=2 k_{F}$, and publish the results elsewhere.

Apparently, there are many features in the $S(\mathbf{q}, \omega)$ spectra, especially the strong q-orientation-dependent fine structure for $q>q_{c}$ and the large and q-orientationdependent width of the spectra for $q<q_{c}$, which must be directly related to band-structure effects and which will be discussed in what follows. In addition to a strong qorientation dependence of the overall shape of the $S(\mathrm{q}, \omega)$ spectra, two features of the spectra, which are visible only for a particular $q$ orientation, and which have already been mentioned in Sec. IV, should be exemplarily interpreted as a lattice-induced fine structure.

\section{B. Energy-gap-induced fine structure of $S(q, \omega)$, pseudopotential calculations}

In Figs. 2-5, the [110] spectra exhibit a secondary peak (shoulder) around $12.5 \mathrm{eV}$ for 0.59 a.u. $\leq q \leq 1.25$ a.u. The dispersion of its peak position $\hbar \omega(q)$, defined by a zero of the third derivative, is given in Fig. 11 together with a plot of the following relation:

$$
\hbar \omega(q)=\left[\hbar^{2} q(2 P-q) / 2 m\right]+E_{g} / 2,
$$

which fits the experiment best with $P=0.88$ a.u. and $E_{g}=4 \mathrm{eV}$.

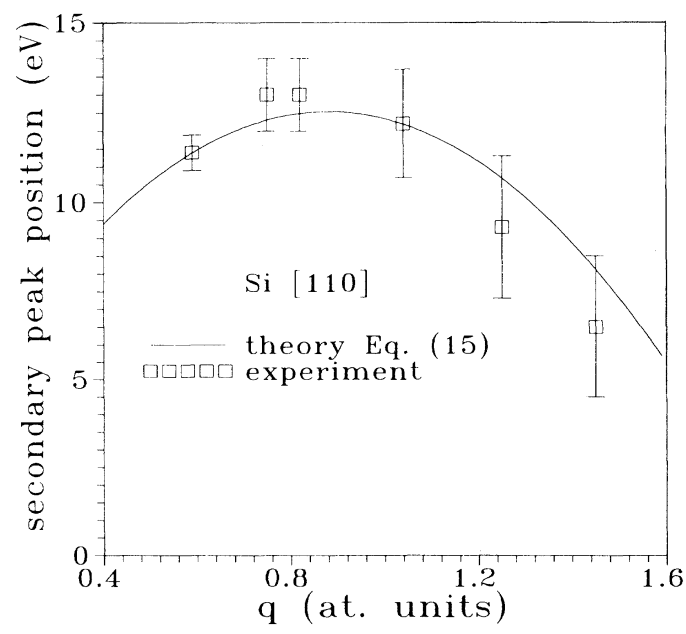

FIG. 11. Squares: Experimental energy position of the secondary peak of $S(\mathbf{q}, \omega)$ for q parallel to [110]. Solid line: Calculated energy position of the secondary peak for $\mathbf{q}$ parallel to [110], according to Eq. (15) with $P=0.86$ a.u.; $E_{g}=4 \mathrm{eV}$.
It has been shown in Ref. 5 that a dispersion relation of this type is a signature for spectral features caused by an enhanced density of those unoccupied states, whose extended-zone-scheme $\mathbf{k}$ vectors end on a plane

$$
\mathbf{P} \cdot \mathbf{k}=P^{2}, \quad \mathbf{P} \| \mathbf{q},
$$

so that the maximum of $\omega(q)$ is obtained for $q=P . E_{g} / 2$ is a constant energy offset, which accounts for an energy gap of magnitude $E_{g}$ on the plane $\mathbf{P} \cdot \mathbf{k}=P^{2}$. Looking at the Si band structure, one easily finds that the enhanced density of states on the 220-type Bragg plane in the extended zone scheme, together with the corresponding Bragg-plane-induced energy gap, can be made responsible for the secondary peak in the [110] spectra. The corresponding states are those of the lowest band of Si between $X$ and $\Gamma$, so that $E_{X}-E_{\Gamma_{0}}\left(E_{\Gamma_{0}}\right.$ is the bottom of the valence band) should determine the maximum-energy loss on the dispersion curve of the secondary peak position. According to a most recent linear muffin-tin orbital (LMTO) band-structure calculation for $\mathrm{Si},{ }^{40} E_{X}-E_{\Gamma_{0}}$ is $13.0 \mathrm{eV}$, which is exactly the maximum experimental peak position $13.0 \mathrm{eV}$ in Fig. 11. Furthermore, the value of $\mathbf{P}$ in Eq. (15), which fits best the experimental peak positions, is very near to $\left|G_{220} / 2\right|=0.865$ a.u. and $E_{g}=4$ $\mathrm{eV}$ is nearby the energy gap at the $X$ point, which is 4.3 $\mathrm{eV}$ in Ref. 40, another strong indication of the validity of the above interpretation.

Finally, we have performed a local-pseudopotential calculation of $S(\mathbf{q}, \omega)$ for $\mathrm{q} \|[110], q=0.59$ a.u., by using the Ehrenreich-Cohen ${ }^{28}$ relation, Eq. (7), for the dielectric function with Brust's ${ }^{41}$ empirical pseudopotential coefficients $V(111)=-0.105$ a.u., $V(220)=0.02$ a.u., and $V(311)=0.04$ a.u. The calculated result has been both exchange corrected according to Eq. (8), and lifetime corrected according to Eq. (9) using the values of the imaginary part of the self-energy of Fig. 10. The result of this calculation is presented in Fig. 12. The secondary

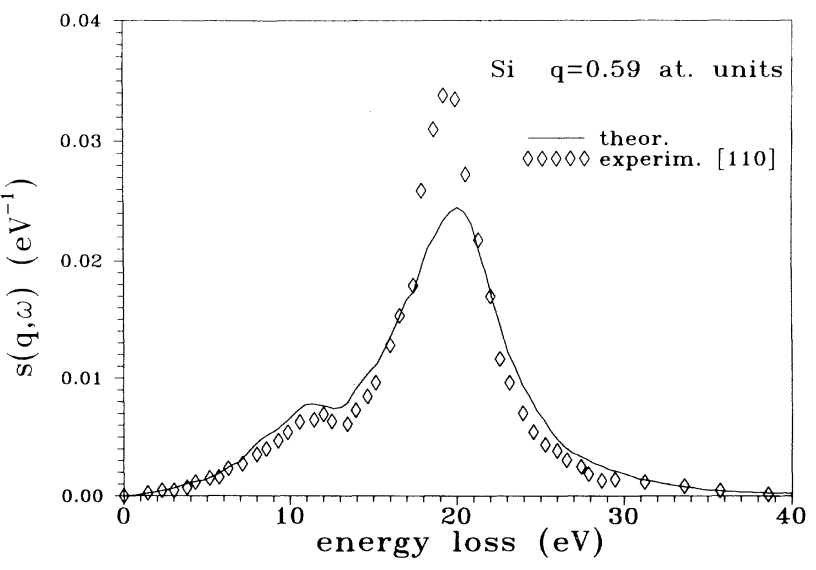

FIG. 12. Rhombs: Experimental $S(\mathbf{q}, \omega)$ for Si for q parallel to [110]; $q=0.59$ a.u. Solid curve: Pseudopotential-calculated $S(\mathbf{q}, \omega)$ of Si for q parallel to [110]; $q=0.59$ a.u. by using $\varepsilon(\mathbf{q}, \omega)$ according to Eq. (7); pseudopotential according to Ref. 41. 
peak at $11.4 \mathrm{eV}$ is well reproduced by this calculation, where its position is insensitive against a variation of the static local-field factor $G(q)$, whereas the position of the main peak depends strongly on $G(q)$. In this calculation we have chosen $G(q)=0.4$ in order to fit the experimental main peak position, where this value of $G(q)$ is nearly three times larger than that given in Ref. 34, but very near the experimental value plotted in Fig. 9. Note that this overcorrection of exchange leads to a much broader main peak compared with experiment. Nevertheless, the good agreement between calculation and experiment with respect to the secondary peak position is again an indication that this feature can be understood quite well in terms of the $\mathrm{Si}$ band structure, where no local-field effects, as represented by Eq. (6), must be invoked.

\section{Plasmon-Fano resonances, pseudopotential calculations}

As already mentioned in Sec. IV, the [111] spectra exhibit a very specific structure for $q \geq 0.59$ a.u., when compared with the [100] spectra. This fine structure becomes especially transparent for $q=1.25$ and 1.45 a.u., where a peak (shoulder) is visible around $14 \mathrm{eV}$ connected with a steep rise around $18 \mathrm{eV}$. For smaller $q$ 's, the much smaller width of the [111] spectra, as compared with the [100] spectra, is evident. But the common origin of these differences only becomes intelligible when the differences [111] - [100] of the normalized spectra are plotted, as shown in Fig. 13. This difference is characterized by a valley-peak structure, with a nearly $q$-independent zero passage between 17.0 and $18.5 \mathrm{eV}$, and an amplitude which is decreasing with increasing $q$. One can also find a similar behavior for the [110]- [111] differences, slightly masked by the energy-gap-induced fine structure of the [110] spectra described in detail in Sec. V B. This way it becomes transparent that the [111] direction plays a special role for the dynamic structure factor of Si. What makes the [111] direction outstanding in Si? It has al-

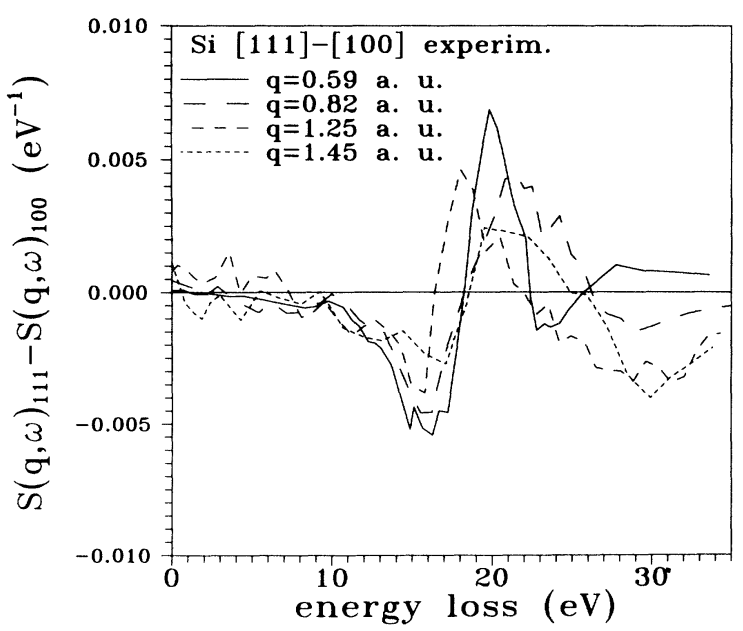

FIG. 13. Experimental differences of $S(\mathbf{q}, \omega)$ curves between [111] and [100] $\mathbf{q}$ orientation for different $q$ values as indicated in the inset. ready been pointed out by Sturm ${ }^{42}$ that the diameter of the first Brillouin zone of $\mathrm{Si}$ in the [111] direction is smaller than $2 q_{c}$, so that higher plasmon bands, as predicted by Saslow and Reiter, ${ }^{25}$ should exist and should be well-defined elementary excitations. But the strong plasmon damping in $\mathrm{Si}$, due to interband transition, prevents a direct experimental verification of the band splitting at the (111) Brillouin-zone boundary, ${ }^{42}$ as can also be seen in Fig. 3. We found no indication of a splitting of the plasmon resonances ${ }^{43}$ for $q=0.59$ a.u., $\mathbf{q} \|[111]$ [this $\mathbf{q}$ is very near the (111) Brillouin-zone boundary], where this splitting should be $1.5 \mathrm{eV} .{ }^{42}$ Moreover, $\rho_{111}$, the $\mathbf{G}_{111}$ th Fourier coefficient of the $\mathrm{Si}$ valence electron density, is nearly one order of magnitude larger than the Fourier coefficients belonging to the next following reciprocal-lattice vectors. ${ }^{44}$ Therefore, umklapp processes, whose strength scales as $\rho_{\mathrm{G}}$, should be visible mainly at the (111) Brillouin-zone boundary, both with respect to the collective plasmon excitation and the continuum of the electron hole excitation, as demonstrated for a two-band model in Fig. 14: On the one hand, plasmon bands with an energy gap at the zone boundary are generated by backfolding the plasmon branch into the first Brillouin zone. Moreover, these plasmon bands become damped by interband transitions, when the electron-hole-excitation continuum is folded back into the first Brillouin zone. This has already been shown by Saslow and Reiter ${ }^{25}$ and by Pandey et al. ${ }^{45}$ On the other hand, as a consequence of the umklapp processes considered by Sturm, Schülke, and Schmitz, ${ }^{11}$ the first plasmon band also continues into the higher zones of the repeated zone scheme, as shown in Fig. 14. This means that the first plasmon band, a discrete excitation, is coupled by the lattice-induced charge fluctuations to the continuum of the electron-hole-pair excitations. According to fundamental relations of coupled quantum-mechanical systems, such a coupling leads to so-called Fano resonances ${ }^{46}$ or antiresonances, whose characteristic is a

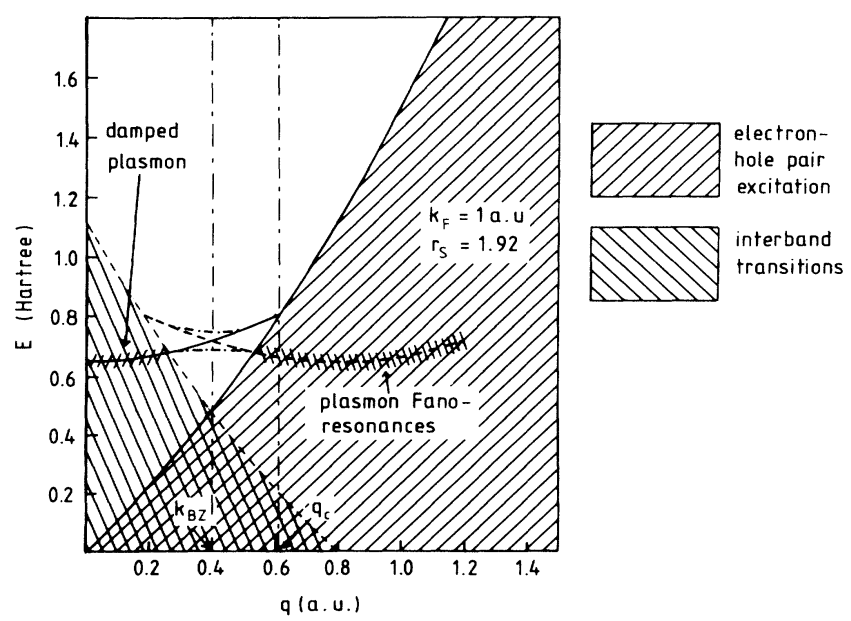

FIG. 14. Illustration of the effect of umklapp processes on the electron-hole-excitation continuum and on the plasmon excitation branch in the repeated zone scheme of a two-plasmonband model. $k_{\mathrm{BZ}}$ is the Brillouin-zone-boundary wave vector. 
valley-peak structure on top of the spectrum of the continuum part of the excitation, where the zero passage of the added part is roughly at the peak position of the discrete excitation. This is exactly what one can see in Fig. 13, where the zero passage of the peak-valley structure is just in the energy range of the first plasmon band, which is shown in Fig. 2. Therefore, it seems justifiable to explain the fine structure found in the [111] spectra, as
plasmon-Fano resonances. This interpretation is additionally supported by calculations ${ }^{11,22}$ of the dynamical structure factor $S(\mathrm{q}, \omega)$ according to Eqs. (4), (5), (6), and (12) within the limits of the so-called two-plasmon-band model, ${ }^{22}$ which means that we neglect all non-diagonal terms in the dielectric matrix of Eq. (6) except $\varepsilon_{\mathrm{G} 0}$ and $\varepsilon_{0 G}$ with $\mathbf{G}=2 \pi / a(1,1,1)$. By doing this, one ends up with the following relation: ${ }^{11}$

$$
S(\mathbf{q}, \omega)=\left(\hbar q^{2} / 4 \pi^{2} e^{2} n\right) \operatorname{Im}\left\{-1 / \varepsilon_{\mathrm{GGcorr}}+\left(\varepsilon_{\mathrm{G} 0 \mathrm{corr}} \varepsilon_{0 \mathrm{Gcorr}} / \varepsilon_{\mathrm{GGcorr}}^{2}\right)\left[-\left(\varepsilon_{\mathrm{G}^{\prime} \mathrm{G}^{\prime \prime} \mathrm{corr}}\right)_{00}^{-1}\right]\right\}[1 /(1-G(q))],
$$

where we have suppressed the arguments $q_{r}$ and $\omega$ in the elements of the dielectric matrix. The first term in the \{ \} brackets represents the short-wavelength electronhole-pair excitations. The second term in the \{\} brackets couples the long-wavelength collective excitations [represented by $\left(\varepsilon_{\mathbf{G}^{\prime} \mathbf{G}^{\prime \prime} \text { corr }}\right)_{00}^{-1}$ ] to the electron-hole-pair excitation continuum, where the complex coupling factor $f_{\mathrm{G}}\left(\mathbf{q}_{r}, \omega\right)=\varepsilon_{\mathrm{G} 0} \varepsilon_{0 \mathrm{G}} / \varepsilon_{\mathrm{GG}}^{2}$ can produce a valley-peak fine structure typical of a Fano resonance, whenever $f_{\mathbf{G}}\left(\mathbf{q}_{r}, \omega\right)$ has a negative imaginary part, since $\operatorname{Re}\left[-\left(\varepsilon_{G^{\prime} G^{\prime \prime} \text { corr }}\right)_{00}^{-1}\right]$ becomes negative for $\omega>\omega_{p}\left(q_{r}\right)$. Additionally, a negative real part of $f_{\mathrm{G}}\left(\mathbf{q}_{r}, \omega\right)$ can account for a plasmon antiresonance due to $\operatorname{Im}\left[-\left(\varepsilon_{G^{\prime} G^{\prime \prime} \text { corr }}\right)_{00}^{-1}\right]$. We have calculated the elements of the dielectric matrix according to Eq. (11) by using the empirical pseudopotential of Brust ${ }^{41}$ (expansion of the pseudo-wave-function into 59 plane waves) thus obtaining $S(\mathrm{q}, \omega)$ by means of Eq. (17) within the limits of the two-plasmon-band model, both for q\|[111] $[\mathbf{G}=(2 \pi / a)(1,1,1)$ in Eq. (17)] and $\mathbf{q} \|[100][\mathbf{G}=$

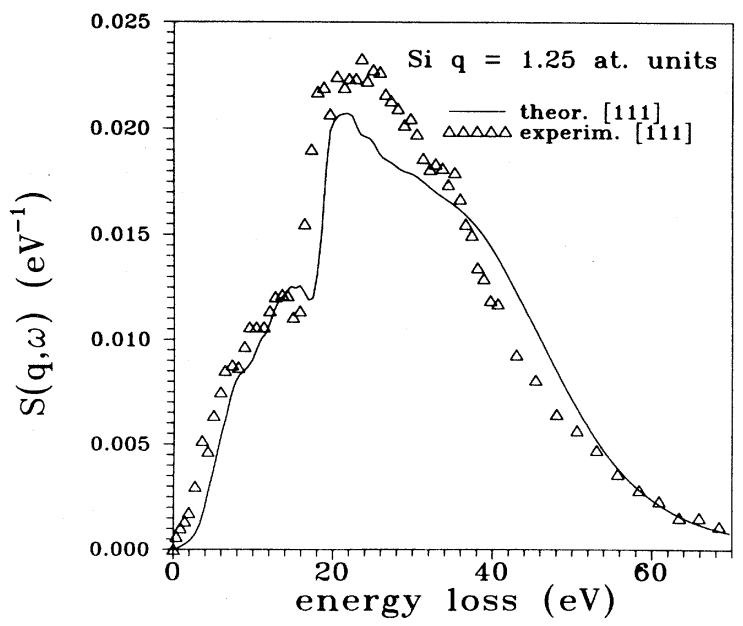

FIG. 15. Triangles: Experimental $S(\mathbf{q}, \omega)$ of $\mathrm{Si}$ for $\mathbf{q}$ parallel to [111]; $q=1.25$ a.u. Solid line: Two-plasmon-band pseudopotential-calculated $S(\mathbf{q}, \omega)$ of $\mathrm{Si}$ for q parallel to [111]; $q=1.25$ a.u. by using $T_{\mathrm{G}^{\prime} \mathrm{G}^{\prime \prime}}$ of Eq. (6); pseudopotential according to Ref. 41 .
$(2 \pi / a)(1,0,0)]$ in each case with $q=1.25$ a.u. The values for the local-field factor $G(q)$ are from Ref. 34. The results of these calculations, convoluted with the experimental resolution, are presented in Figs. 15 and 16, together with the corresponding nonsmoothed experimental spectra. In Fig. 15, the structure typical for plasmonFano resonances is well reproduced by the calculations, although its position on the energy scale is shifted by roughly $2 \mathrm{eV}$ to larger energy losses when compared with experiment. As already stated in connection with Fig. 12, the dispersion of the plasmon resonance are not exactly restored by the local-pseudopotential calculations. Nevertheless, one can easily verify that the two-plasmon-band model can account, on the one hand, for the appearance of the plasmon-Fano-resonance structure for $q \|[111]$, due to the large value of $\rho_{111}$ (see Fig. 15), and, on the other hand, for the absence of this structure for $q \|[100]$, due to the zero of $\rho_{200}$ for the diamond structure (see Fig. 16). We have already shown in Fig. 13, where the experimental differences between the $\mathbf{q} \|[111]$ and the $\mathbf{q} \|[100]$

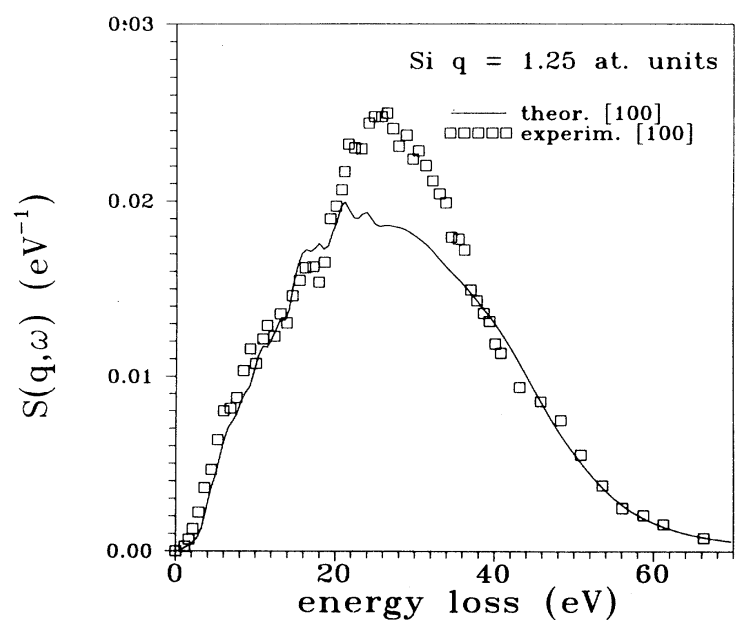

FIG. 16. Squares: Experimental $S(\mathbf{q}, \omega)$ of $\mathrm{Si}$ for $\mathrm{q}$ parallel to [100]; $q=1.25$ a.u. Solid line: Two-plasmon-band pseudopotential-calculated $S(\mathbf{q}, \omega)$ of $\mathrm{Si}$ for q parallel to [100]; $q=1.25$ a.u., by using $T_{\mathrm{G}^{\prime} \mathrm{G}^{\prime \prime}}$ of Eq. (6); pseudopotential according to Ref. 41. 
spectra were plotted, that the appearance of plasmonFano resonances in the experiment is not restricted to $q=1.25$ a.u., but is found in the $q \|[111]$ spectra for the whole range $G_{111} / 2<q<3 G_{111} / 2$ (see Fig. 14), even if the typical valley-peak structure is not directly recognizable. This experimental finding is nicely proved by corresponding calculations of the above differences, as shown in Fig. 17, at least qualitatively: for all $q$ 's within the above range, the calculations, performed within the limits of the two-plasmon-band model, account for a valleypeak structure typical of Fano resonances. Moreover, the agreement between experiment and calculation is not only qualitatively. Thus the experimental $q$ dependence of the relative peak position of the peak strength and of the zero passages are well reproduced by the calculation, even though the absolute position of the zero passages are at lower-energy losses in the experiment by roughly $2 \mathrm{eV}$, as already commented upon in connection with Fig. 15.

\section{Static structure factor, jellium model calculations}

In Fig. 4, we confront the experimental values of the static structure factor $S(\mathrm{q})$ with calculations according to the different approaches within the limit of the jellium model, as already partly discussed in Sec. V A. The Hartree-Fock model completely neglects dielectric screening, and takes into account only Pauli's exclusion principle, so that one has to insert the following jellium response function $\varepsilon^{-1}(\mathbf{q}, \omega)$ into Eq. (4):

$$
\varepsilon^{-1}(\mathbf{q}, \omega)=1+\lim _{\eta \rightarrow 0}\left(4 \pi e^{2} / q^{2}\right) \sum_{\mathbf{p}}\left[f_{0}(\mathbf{p}+\mathbf{q})-f_{0}(\mathbf{p})\right] /[E(\mathbf{p}+\mathbf{q})-E(\mathbf{p})-\hbar \omega+i \eta],
$$

where $E(\mathbf{p})$ and $f_{0}(\mathbf{p})$ are the energy and occupation number, respectively, of the momentum eigenstate $|p\rangle$. One can easily verify that this approximation is far from the experiment. The corresponding Hartree-Fock radial pair-correlation function $g(r)$, extracted from $S(q)$ via

$g(r)=\left(1 / 2 n \pi^{2}\right) \int[S(q)-1][\sin (q r) / q r] q^{2} d q$

and shown in Fig. 18, reflects the repulsive action of Pauli's principle for electrons with parallel spins together with the complete neglection of Coulomb correlation. Especially for smaller $q$ 's the RPA $S(q)$ much better fits the experiment, whereas for $k_{F}<q<2 k_{F}$ only the exchange-corrected jellium $S(q)$ satisfactorily approaches the experiment, at least its orientation average. The corresponding pair-correlation functions are shown again Fig. 18. The large negative part of $g(r)$ for the RPA is

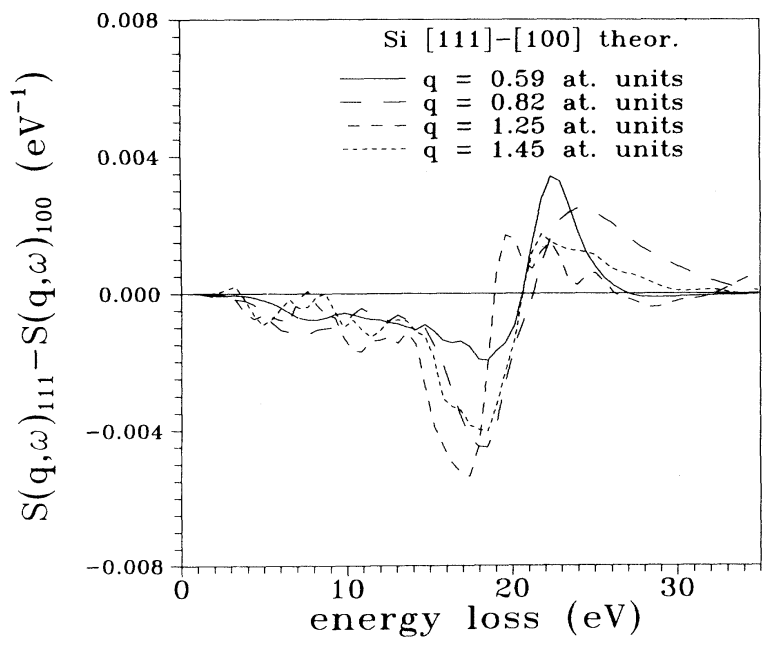

FIG. 17. Calculated differences of $S(\mathbf{q}, \omega)$ curves between [111] and [100] $q$ orientation for different values of $q$, as indicated in the inset. consistent with deviations of the RPA from experiment for $q>k_{F}$. As already mentioned in Secs. III and IV, the onset of the core contribution around $70 \mathrm{eV}$ prevents reliable values of $S(q)$ for $q>2 k_{F}$, so that the question whether there exists a somewhat oscillatory behavior of $S(q)$ must be left unanswered.

\section{CONCLUSIONS}

The comparison of the experimental dynamic and static structure factor for electrons in Si single crystals with various theoretical models has led to the following conclusions.

(i) The plasmon resonances of Si shown by the measured dynamic structure factor for $q<q_{c}$ are much more damped by interband transitions than corresponding

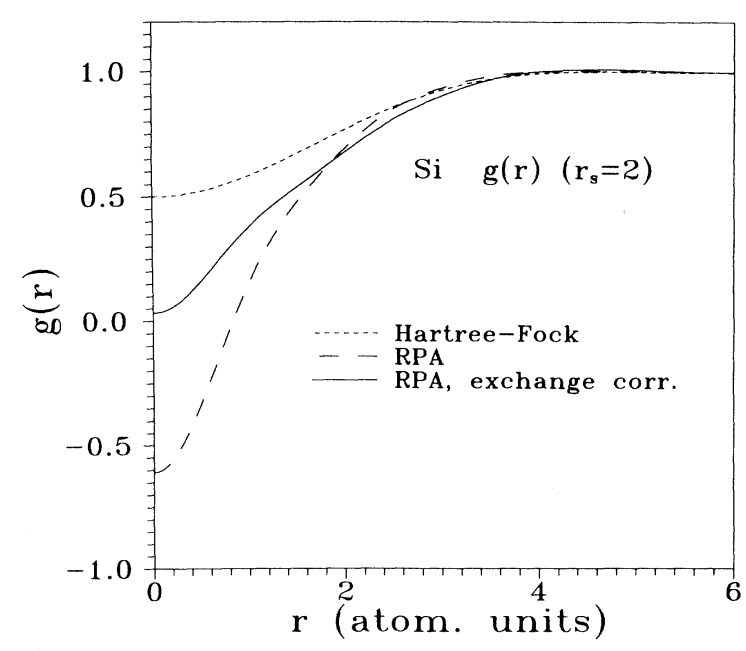

FIG. 18. Pair-correlation function $g(r)$ obtained from jellium-model-calculated static structure factors of Fig. 7 for different approximations, as indicated in the inset. 
features in the case of simple metals.

(ii) As already stated for simple metals, and also in the case of $\mathrm{Si}$, the Hartree-Fock approximation and the RPA cannot appropriately describe the gross features of the short-range correlation of electrons, as measured with the dynamic structure factor for $q>q_{c}$.

(iii) Only by taking into account both the exchange correction to the dielectric functions via a static so-called local-field factor, and lifetime effects via the on-shell approximation of the imaginary part of the self-energy, the short-range electron correlation can be appropriately considered, so that the overall features (peak position and dispersion, width, and shape) of the orientation averaged $S(\mathrm{q}, \omega)$ 's and the $q$ dependence of the orientationaveraged $S(\mathrm{q})$ 's are correctly represented. A fitting of the local-field factor to the experiment seems to be reasonable.

(iv) The dynamic structure factor of $\mathrm{Si}$ exhibits a much stronger q-orientation dependence of the peak position, width, and shape of the spectra than the simple metals investigated so far. Some of the q-orientation-dependent fine structure can be understood as being directly related to an enhanced density of states at zone boundaries in the extended zone scheme, in good agreement with pseudopotential band-structure calculations.

(v) As a consequence of ion-lattice-induced local fields, a volume plasmon-band-structure arises, which should be especially pronounced for $q \|[111]$, since in that direction $q_{c}$ is smaller than the half-diameter of the Brillouin zone. However, direct evidence of the plasmon-band splitting is prevented by the strong interband damping of the plasmon resonances. But it could clearly be demonstrated that, as a consequence of the plasmon-band structure, the coupling of the electron-hole-pair excitation continuum to the discrete plasmon excitations leads to plasmonFano resonances, whose existence could be experimentally proved and whose characteristics could be well described within the limits of a two-plasmon-band model.

\section{ACKNOWLEDGMENTS}

This work was funded by the German Federal Ministry of Research and Technology under Contract No. 05-434 AXB. Many discussions with K. Sturm were extremely helpful for the interpretation of the experiment.
${ }^{1}$ W. Schülke, H. Nagasawa, and S. Mourikis, Phys. Rev. Lett. 52, 2065 (1984).

${ }^{2}$ W. Schülke, H. Nagasawa, S. Mourikis, and P. Lanzki, Phys. Rev. B 33, 6744 (1986).

${ }^{3}$ W. Schülke, A. Berthold, A. Kaprolat, and H.-J. Güntherodt, Phys. Rev. Lett. 60, 2217 (1988).

${ }^{4}$ W. Schülke, U. Bonse, H. Nagasawa, A. Kaprolat, and A. Berthold, Phys. Rev. B 38, 2112 (1988).

${ }^{5}$ W. Schülke, H. Nagasawa, S. Mourikis, and A. Kaprolat, Phys. Rev. B 40, 12215 (1989).

${ }^{6}$ W. Schülke, H. Schulte-Schrepping, and J. R. Schmitz, Phys. Rev. B 47, 12426 (1993).

${ }^{7}$ D. M. Miliotis, Phys. Rev. B 3, 701 (1971).

${ }^{8}$ P. Eisenberger, P. M. Platzman, and K. C. Pandy, Phys. Rev. Lett. 31, 311 (1973); P. M. Platzman and P. Eisenberger, ibid. 33, 152 (1974); P. Eisenberger and P. M. Platzman, Phys. Rev. B 13, 934 (1976).

${ }^{9}$ W. Schülke and W. Lautner, Phys. Status Solidi B 66, 211 (1974).

${ }^{10}$ W. Schülke, U. Bonse, H. Nagasawa, S. Mourikis, and A. Kaprolat, Phys. Rev. Lett 59, 1361 (1987).

${ }^{11}$ K. Sturm, W. Schülke, and J. R. Schmitz, Phys. Rev. Lett. 68, 228 (1992).

${ }^{12}$ W. Schülke, Phys. Lett. 83A, 451 (1981).

${ }^{13}$ W. Schülke, Solid State Commun. 43, 863 (1982).

${ }^{14}$ A. Kaprolat and W. Schülke, Z. Naturforsch 48a, 227 (1993).

${ }^{15}$ W. Schülke and A. Kaprolat, Phys. Rev. Lett. 67, 879 (1991).

${ }^{16}$ K. Sturm and W. Schülke, Phys. Rev. B 46, 7193 (1992).

${ }^{17}$ P. Zacharias, J. Phys. C 7, L26 (1974); J. Phys. F 5, 645 (1975).

${ }^{18}$ J. Stiebling and H. Raether, Phys. Rev. Lett. 40, 1293 (1978).

${ }^{19}$ C. H. Chen, A. E. Meixner, and B. M. Kincaid, Phys. Rev. Lett. 44, 951 (1980).

${ }^{20}$ J. P. Walter and M. L. Cohen, Phys. Rev. B 5, 3101 (1972).

${ }^{21}$ S. G. Louie, J. R. Chelikowsky, and M. L. Cohen, Phys. Rev. Lett. 34, 155 (1975).

${ }^{22}$ K. Sturm and L. E. Oliveira, Phys. Rev. B 22, 6268 (1980); L.
E. Oliveira and K. Sturm, ibid. 22, 6283 (1980)

${ }^{23}$ S. L. Adler, Phys. Rev. 126, 413 (1962).

${ }^{24}$ N. Wiser, Phys. Rev. 129, 62 (1963).

${ }^{25}$ W. M. Saslow and G. F. Reiter, Phys. Rev. B 7, 2995 (1973).

${ }^{26}$ P. Nozieres and D. Pines, Phys. Rev. 113, 1254 (1959).

${ }^{27}$ D. Pines and P. Nozieres, The Theory of Quantum Liquids (Benjamin, New York, 1966), Vol. 1.

${ }^{28}$ H. Ehrenreich and M. H. Cohen, Phys. Rev. 115, 786 (1959).

${ }^{29}$ J. J. Quinn and R. A. Terrell, Phys. Rev. 112, 812 (1958).

${ }^{30}$ S. Rahman and G. Vignale, Phys. Rev. B 30, 6951 (1984).

${ }^{31}$ T. K. Ng and B. Dabrowski, Phys. Rev. B 33, 5358 (1986).

${ }^{32}$ N. D. Mermin, Phys. Rev. B 1, 2362 (1970).

${ }^{33}$ J. Hubbard, Proc. R. Soc. London Ser. A 243, 336 (1957).

${ }^{34}$ S. Ichimaru and K. Utsumi, Phys. Rev. B 24, 7385 (1981)

${ }^{35}$ A. Berthold, S. Mourikis, J. R. Schmitz, W. Schülke, and H. Schulte-Schrepping, Nucl. Instrum Methods Phys. Res. Sect. A 317, 373 (1992).

${ }^{36}$ C. J. Sparks, G. E. Ice, J. Wong, and B. W. Batterman, Nucl. Instrum. Methods 172, 237 (1980).

${ }^{37}$ W. Schülke, Nucl. Instrum. Methods Phys. Res. Sect. A 246, 491 (1986).

${ }^{38}$ C. Reinsch, Numer. Mathematik 10, 177 (1967); 16, 451 (1971).

${ }^{39}$ F. Brosens, J. T. Devrese, and L. F. Lemmens, Phys. Rev. B 21, 1363 (1980).

${ }^{40}$ N. E. Christensen (private communication).

${ }^{41}$ D. Brust, Phys. Rev. 134, A1337 (1964).

${ }^{42}$ K. Sturm, Adv. Phys. 31, 1 (1982).

${ }^{43}$ It has been shown in Ref. 15 that one can obtain experimental evidence of the existence of volume plasmon bands by utilizing coherent inelastic scattering.

${ }^{44}$ W. Schülke and S. Mourikis, Acta Crystallogr. Sec. A 42, 86 (1986).

${ }^{45}$ K. C. Pandey, P. M. Platzman, P. Eisenberger, and E-Ni Foo, Phys. Rev. B 9, 5046 (1974).

${ }^{46}$ U. Fano, Phys. Rev. 124, 1866 (1961). 